PROCEEDINGS OF THE

AMERICAN MATHEMATICAL SOCIETY

Volume 130, Number 7 , Pages 1937-1941

S 0002-9939(02)06300-1

Article electronically published on February 12, 2002

\title{
ISOMORPHISM OF COMMUTATIVE GROUP ALGEBRAS OF CLOSED $p$-GROUPS AND $p$-LOCAL ALGEBRAICALLY COMPACT GROUPS
}

\author{
PETER DANCHEV
}

(Communicated by Stephen D. Smith)

\begin{abstract}
Let $G$ be an abelian group and let $K$ be a field of $\operatorname{char} K=p>0$. It is shown via a universal algorithm that if the modified Direct-Factor Problem holds, then the $K$-isomorphism $K H \cong K G$ for some group $H$ yields $H \cong G$ provided $G$ is a closed $p$-group or a $p$-local algebraically compact group. In particular, this is the case when $G$ is closed $p$-primary of arbitrary power, or $G$ is $p$-local algebraically compact with cardinality at most $\aleph_{1}$ and $K$ is in cardinality not exceeding $\aleph_{1}$. The last claim completely settles a question raised by W. May in Proc. Amer. Math. Soc. (1979) and partially extends our results published in Rend. Sem. Mat. Univ. Padova (1999) and Southeast Asian Bull. Math. (2001).
\end{abstract}

\section{INTRODUCTION}

In 1979, Warren May in 11] asked if a p-torsion abelian group being closed (in other words, torsion-complete) is an invariant property for the commutative group algebra of this group over an arbitrary field with characteristic $p$. We gave a partial answer in [2, 4] to this problem by restricting the field to have cardinality $p$ and the group to have cardinality less than or equal to the first uncountable cardinal $\aleph_{1}$ (actually, we have established stronger affirmations). Here we shall develop our mixed algebraic-topological technique which generalizes the above-cited fact for a field with characteristic $p$ of arbitrary power and which gives new perspectives for an investigation of a dual class of abelian groups called algebraically compact groups. For this purpose we first formulate the following well-known and long-standing question, namely

Direct-Factor Problem. Does it follow that the $p$-component $G_{p}$ of the abelian group $G$ is a direct factor of the normed Sylow p-subgroup $S(K G)$ in the group algebra $K G$ over a field $K$ of characteristic $p$ ? As a consequence, does it follow that the p-mixed abelian group $G$ is a direct factor of the group $V(K G)$ of all normalized units?

Additional information, however, ensures the next crucial modification obtained in a general form from May (cf. [12]).

Received by the editors May 19, 2000 and, in revised form, February 5, 2001.

2000 Mathematics Subject Classification. Primary 20C07; Secondary 20K10, 20K20, 20 K21.

Key words and phrases. Group algebras, isomorphism, closed p-groups, $p$-local algebraically compact groups. 
Modified Direct-Factor Problem. For $G$ an abelian group and $K$ a perfect field with char $K=p>0$ is it true that $S(K G) / G_{p}$ is totally projective? As a consequence, is $V(K G) / G$ then totally projective whenever $G$ is p-mixed?

In [5] we have proved (about this see also [1]) that the Direct-Factor Problem is valid in the case when $G$ is a direct sum of closed $p$-groups or is a direct sum of $p$-local algebraically compact groups; the complementary factors are not described.

\section{MAIN RESULT}

We come now to the main goal that motivates this article, namely

Central Theorem. Suppose $G$ is a closed abelian p-group or a p-local algebraically compact abelian group and $K$ is a field of characteristic $p>0$. If the Modified Direct-Factor Problem is fulfilled, then $K H \cong K G$ as $K$-algebras for any group $H$ if and only if $H \cong G$.

Proof. Without loss of generality we presume that $K G=K H$ and that $K$ is perfect. Further we consider two cases, namely

Case 1. $G$ is p-torsion closed. So, owing to the Modified Direct-Factor Hypothesis we may write $V(K G) \cong G \times V(K G) / G \cong H \times V(K H) / H \cong V(K H)$, where $V(K G) / G$ must be totally projective. On the other hand the separability of $G$ trivially yields that $V(K G)$ is so, hence the same is valid for $V(K G) / G$ as a "subgroup". Therefore from [9], $V(K G) / G$ is a direct sum of cyclics and hence according to [10], $H$ is itself semi-closed as a direct factor of the semi-closed $V(K G)$. Thus, we can write $H=T \times C$, where $T$ is closed and $C$ is a direct sum of cyclics. But $C$ may be represented as a countable ascending union of pure and bounded subgroups, i.e. in other words we write $C=\bigcup_{n} C_{n}, C_{n} \subseteq C_{n+1}$ are pure in $C$ and $C_{n}^{p^{n}}=1$. Consequently, we derive $H=\bigcup_{n} H_{n}$, where $H_{n}=T \times C_{n} \subseteq H_{n+1}$ are pure closed subgroups in $H$. Furthermore $V(K G)=V(K H)=\bigcup_{n} V\left(K H_{n}\right)$ hence $G=\bigcup_{n}\left[V\left(K H_{n}\right) \cap G\right]$.

Now, we select a special sequence $\left(g_{n}\right) \in G$ with the properties: $g_{n}=\alpha_{1}^{(n)} h_{1}^{(n)}+$ $\cdots+\alpha_{s_{n}}^{(n)} h_{s_{n}}^{(n)}$, where $\alpha_{1}^{(n)}, \ldots, \alpha_{s_{n}}^{(n)} \in K$ and $\alpha_{1}^{(n)}+\cdots+\alpha_{s_{n}}^{(n)}=1 ; h_{1}^{(n)}=a_{1}^{(n)} c_{1}^{(n)} \in$ $T \times C_{n}, \ldots, h_{s_{n}}^{(n)}=a_{s_{n}}^{(n)} c_{s_{n}}^{(n)} \in T \times C_{n}, c_{1}^{(n)} \neq \cdots \neq c_{s_{n}}^{(n)} \neq c_{1}^{(n)}$ and $\operatorname{order}\left(c_{r}^{(n)}\right)=$ $\cdots=\operatorname{order}\left(c_{s_{n}}^{(n)}\right)=p^{n}$ for some natural number $r$ with $r \leq s_{n}, s_{n} \in N$, such that $\left(g_{n}\right)$ is a bounded, say $g_{n}^{p}=1$, Cauchy sequence but $\left(h_{r}^{(n)}\right), \ldots,\left(h_{s_{n}}^{(n)}\right)$ are not "a priori" bounded however. Now, we make the following choice: $h_{1}^{(n)^{p}}=\cdots=$ $h_{r-1}^{(n)^{p}}=1, \alpha_{1}^{(n)}+\cdots+\alpha_{r-1}^{(n)}=1 ; h_{r}^{(n)^{p}}=\cdots=h_{s_{n}}^{(n)^{p}} \neq 1, \alpha_{r}^{(n)}+\cdots+\alpha_{s_{n}}^{(n)}=0$ and

$$
\begin{aligned}
& \alpha_{1}^{(n+L)} h_{1}^{(n+L)}+\cdots+\alpha_{s_{n+L}}^{(n+L)} h_{s_{n+L}}^{(n+L)} \\
& \quad=\left[\alpha_{1}^{(n)} h_{1}^{(n)}+\cdots+\alpha_{s_{n}}^{(n)} h_{s_{n}}^{(n)}\right]\left(\beta_{1}^{(n)} a_{1}^{(n)}+\cdots+\beta_{m_{n}}^{(n)} a_{m_{n}}^{(n)}\right)
\end{aligned}
$$

for some natural number $m_{n}$ and each integer $L \geq 0$ where $a_{1}^{(n)} \in H^{p^{n}}, \ldots, a_{m_{n}}^{(n)} \in$ $H^{p^{n}}$, hence $\beta_{1}^{(n)} a_{1}^{(n)}+\cdots+\beta_{m_{n}}^{(n)} a_{m_{n}}^{(n)} \in V^{p^{n}}(K H)=V^{p^{n}}(K G)$ and so $g_{n+L} g_{n}^{-1} \in$ $G \cap V^{p^{n}}(K G)=G^{p^{n}}$. Evidently, the choice of such a sequence is possible only when such $s_{n} \geq 3$ exists. If so, we observe further that Kulikov's criterion ([9], p. 38, Theorem 70.7) implies $\left(g_{n}\right)$ converges to an element of $G$. Certainly the boundary is of the form $f_{1} b_{1}^{(j)}+f_{2} b_{2}^{(j)}+\cdots+f_{t} b_{t}^{(j)} \neq 1$ for a fixed $t \in N$ and a fixed $j \in N$ 
so that $b_{1}^{(j)}, \ldots, b_{t}^{(j)} \in H_{j}$ and $f_{1}, \ldots, f_{t} \in K$ with $f_{1}+\cdots+f_{t}=1$. Since

$$
f_{1} b_{1}^{(j)}+\cdots+f_{t} b_{t}^{(j)} \in\left[\alpha_{1}^{(n)} h_{1}^{(n)}+\cdots+\alpha_{s_{n}}^{(n)} h_{s_{n}}^{(n)}\right] G^{p^{k}}
$$

for every $k \geq 1$ and every $n \geq k$, the above representation of $G$ as a special countable union of groups yields a fixed element $1 \neq c^{(j)} \in C_{j}$ and some $c_{m}^{(n)} \in C_{n}$ for $r \leq m \leq s_{n}$ with the property $c^{(j)} \in c_{m}^{(n)} C^{p^{n}}$. But when $n>j$ we get $c^{(j)} c_{m}^{(n)^{-1}} \in C^{p^{n}} \cap C_{n}=1$, hence $c^{(j)}=c_{m}^{(n)}$, a contradiction. Moreover, eventually $h_{i}^{(n)} \in h_{m}^{(n)} G^{p^{k}}$ for each $k \geq 1, n \geq k$ and some $1 \leq i \neq m \leq s_{n}$, whence $c_{i}^{(n)} \in c_{m}^{(n)} C^{p^{k}}$ and thus $c^{(j)}=c_{i}^{(n)}$ for every positive integer $n>j$. The same procedure works and for the other nonidentity elements of $C_{j}$ from the boundary. Consequently $C$ must be bounded. That is, by [9], $H$ must be closed, as claimed.

Now if each bounded Cauchy sequence $\left(g_{n}\right)$ in $G$ is represented as $g_{n}=\alpha_{n} h_{n}=$ $h_{n}$ where $1=\alpha_{n} \in K$ and $h_{n} \in H$ or $g_{n}=\alpha_{1}^{(n)} h_{1}^{(n)}+\alpha_{2}^{(n)} h_{2}^{(n)}$ where $\alpha_{1}^{(n)}, \alpha_{2}^{(n)} \in K$ with $\alpha_{1}^{(n)}+\alpha_{2}^{(n)}=1$ and $h_{1}^{(n)}, h_{2}^{(n)} \in H$, then it follows that every bounded Cauchy sequence of $H$ is also of the two types presented and thus it holds obviously that an arbitrary restricted Cauchy sequence in $H$ possesses a stabilization in $H$ for almost all natural numbers, i.e. it is convergent in $H$. Furthermore, the above-mentioned Kulikov's theorem implies that $H$ is itself closed, as stated.

Finally, in all cases, $G \cong H$ because the group Ulm-Kaplansky functions are invariants of the commutative modular group algebra (cf. [11]) and determine up to an isomorphism the closed $p$-groups (see for example [9] or [11]).

Case 2. $G$ is p-local algebraically compact. By [3], we may assume that $G$ and $H$ are reduced, and the $p$-locality of $H$ follows trivially. Moreover applying the Modified Direct-Factor Conjecture we deduce as above that $G \times V(F G) / G \cong$ $H \times V(F H) / H$, where $V(F G) / G$ is totally projective $p$-torsion. But $G$ algebraically compact does imply that $G_{p}$ is separable (see 9]) whence the same is true for $S(F G) / G_{p} \cong V(F G) / G$ owing to the fact that $G_{p}$ is nice in $S(F G)$ (for instance see [2, 12]). Consequently $V(F G) / G$ is a direct sum of $p$-cyclics. Thus by similar arguments to [10], $H$ can be written as $H=A \times C$, where $A$ is $p$-local algebraically compact and $C$ is a direct sum of $p$-cyclics. That is why analogous to the above $C=\bigcup_{n} C_{n}, C_{n} \subseteq C_{n+1}$ are pure in $C$ and $C_{n}^{p^{n}}=1$. Furthermore $H=\bigcup_{n} H_{n}$, where $H_{n}=A \times C_{n} \subseteq H_{n+1}$ are pure algebraically compact subgroups in $H$. On the other hand $V(K G)=V(K H)=\bigcup_{n} V\left(K H_{n}\right)$ hence $G=\bigcup_{n}\left[V\left(K H_{n}\right) \cap G\right]$. After this, choose the same special sequence $\left(g_{n}\right)$ defined as in Case 1 with the exception that $g_{n}$ need not be bounded. Complying with the Kaplansky criterion (9], p. 191, Theorem 39.1) we may derive that $\left(g_{n}\right)$ is convergent to an element of $G$. In the spirit of the situation of similar reasons as above, $C$ must be bounded. Finally we can conclude that $H$ is algebraically compact $p$-local too (see 9]), and thus from an application of 3 it follows that $G$ and $H$ are isomorphic, as well. The proof is finished after all.

\section{Applichtions}

The first actual consequence of the major theorem is the following assertion announced in [7, 8].

Corollary. Let $G$ be a closed abelian $p$-group and let $K$ be a field with char $K=$ $p \neq 0$. Then for some group $H$ it is true that $K H \cong K G$ as $K$-algebras if and only if $H \cong G$. 
Proof. Since length $(G)=$ length $(H) \leq \omega$, the proof follows automatically by combining the main result in [6] concerning the Direct-Factor Conjecture along with our Central Theorem.

Remark. The attainment above completely solves a problem of May [11] posed in these Proceedings in 1979.

In this direction, we can formulate and prove

Corollary. Let $G$ be an algebraically compact $p$-local abelian group and let $K$ be a field of $\operatorname{char} K=p \neq 0$ both with powers at most $\aleph_{1}$. Then for some group $H$ it is valid that $K H \cong K G$ as $K$-algebras if and only if $H \cong G$.

Proof. Since $|G|=|H| \leq \aleph_{1}$ and length $\left(G_{p}\right)=\operatorname{length}\left(H_{p}\right) \leq \omega$, by employing our Direct-Factor Theorem in 4 plus the Central Theorem argued above we are done.

The following commentary is valuable.

Remark. An example of a closed $p$-group with power $\aleph_{1}$ is given by us in 2 provided that the Continuum Hypothesis holds. By the same token we may construct an abelian closed $p$-primary group of arbitrary cardinality $\aleph_{\alpha}$ where $\alpha$ is any ordinal. Besides, the above corollaries were proved in 2, 4, where $K$ is only the field of $p$ elements (i.e. the simple hence finite field in characteristic $p$ ) and $G$ is of cardinality $\aleph_{1}$, but using an algebraic technique and more especially the so-called back-andforth method together with the Direct-Factor Conjecture.

A problem of some interest and importance in abelian group theory is to determine the criterion illustrated when one countable ascending union of abelian groups can be a closed $p$-group or a $p$-mixed algebraically compact group, respectively. Such a necessary and sufficient condition will be useful for another new and elegant proof of the Central Theorem.

\section{ACKNOWLEDGMENTS}

The author is very grateful to the expert referee for the warm comments and valuable suggestions so needed for improving the original manuscript and the presentation. I would also like to thank the Editor Professor Stephen D. Smith for his advice, and Mrs. Mary Letourneau from the Editorial Department of the AMS for her precise help and control in typesetting this research paper.

\section{REFERENCES}

[1] P. Danchev, Normed unit groups and direct factor problem for commutative modular group algebras, Math. Balkanica, (2-3) 10 (1996), 161-173. MR 98m:16038

[2] - Isomorphism of modular group algebras of direct sums of torsion-complete abelian p-groups, Rend. Sem. Mat. Univ. Padova, 101 (1999), 51-58. MR 2001i:16053

[3] _ Invariants for group algebras of abelian groups, Rend. Circolo Mat. Palermo, to appear.

[4] Commutative group algebras of cardinality $\aleph_{1}$, Southeast Asian Bull. Math., (4) 25 (2001).

[5] - The splitting problem and the direct factor problem in modular abelian group algebras, Math. Balkanica, (3-4) 14 (2000), 217-226.

[6] Commutative group algebras of direct sums of $\sigma$-summable abelian p-groups, Math. J. Okayama Univ., in press.

[7] - Isomorphism of commutative group algebras of mixed splitting groups, Compt. Rend. Acad. Bulg. Sci., (1-2) 51 (1998), 13-16. CMP 2000:02 
[8] - Invariances in commutative and noncommutative group algebras, Compt. Rend. Acad. Bulg. Sci., (4) 54 (2001), 5-8. CMP 2001:11

[9] L. Fuchs, Infinite Abelian Groups I and II, Mir, Moscow, 1974 and 1977. MR 41:333; MR 50:2362

[10] J. Irwin, F. Richman and E. Walker, Countable direct sums of torsion complete groups, Proc. Amer. Math. Soc., 17 (1966), 763-766. MR 33:5721

[11] W. May, Modular group algebras of totally projective p-primary groups, Proc. Amer. Math. Soc., (1) 76 (1979), 31-34. MR 80j:20011

[12] _ The direct factor problem for modular abelian group algebras, Contemp. Math., 93 (1989), 303-308. MR 90c:20071

Department of Mathematics, Plovdiv State University, 4000 Plovdiv, Bulgaria - and - Insurance Supervision Directorate, Ministry of Finance, 1000 Sofia, Bulgaria

Current address: 13 General Kutuzov Street, bl. 7, floor 2, flat 4, 4003 Plovdiv, Bulgaria

E-mail address: peter_v@bulstrad.bg

E-mail address: library@math.bas.bg 